\title{
Genotype-phenotype correlation in 27 pediatric patients in congenital adrenal hyperplasia due to 21-hydroxylase deficiency in a single center
}

\author{
Yangho Yoo, MD', \\ Mi Sun Chang, MD', \\ Jieun Lee, MD', \\ Sung Yoon Cho, $\mathrm{MD}^{2}$, \\ Sung Won Park, MD', \\ Dong-Kyu Jin, MD, PhD', \\ Hyung-Doo Park, MD, PhD ${ }^{4}$ \\ 'Department of Pediatrics, Samsung \\ Medical Center, Sungkyunkwan \\ University School of Medicine, \\ Seoul, ${ }^{2}$ Department of Pediatrics, \\ Hanyang University Guri Hopistal, \\ Hanyang University College of \\ Medicine, Guri, ${ }^{3}$ Department of \\ Pediatrics, Cheil General Hospital \\ \& Woman's Healthcare Center, \\ Kwandong University College of \\ Medicine, Seoul, ${ }^{4}$ Department \\ of Laboratory Medicine and \\ Genetics, Samsung Medical Center, \\ Sungkyunkwan University School \\ of Medicine, Seoul, Korea
}

Received: 30 August, 2013 Revised: 16 September, 2013 Accepted: 30 September, 2013

Address for correspondence: Dong-Kyu Jin, MD, PhD

Department of Pediatrics, Samsung Medical Center, Sungkyunkwan University School of Medicine, 81 Irwon-ro, Gangnam-gu,

Seoul 135-710, Korea

Tel: +82-2-3410-3525

Fax: +82-2-3410-0043

E-mail:jindk@skku.edu
Purpose: The purpose of the study was to evaluate endocrine patterns of patients with congenital adrenal hyperplasia and each gene mutation and to analyze the correlation between each phenotype and genotype.

Methods: This was a retrospective study of the patients with congenital adrenal hyperplasia in the pediatric outpatient clinic at the Samsung Medical Center from November 1994 to December 2012. We analyzed the medical records of 27 patients (male, 19; female, 8) with congenital adrenal hyperplasia who had been diagnosed by genetic testing to have 21-hydroxylase deficiency.

Results: In genetic analysis of 54 alleles from 27 patients, 13 types of mutations were identified. The distribution of 21-hydroxylase deficiency gene mutations revealed that intron 2 splice site (c.293-13A/C > G ) mutations and large deletions were the most common, at $31.5 \%$ and $22.2 \%$ respectively, followed by p.1173N, p.R356W, and p. $.172 \mathrm{~N}$ mutations at $11.1 \%, 9.3 \%$, and $9.3 \%$, respectively. Other mutations were observed at 1.9-3.7\%. No novel mutations were detected

Conclusion: The analysis of 54 alleles revealed 13 types of mutation. The salt wasting form showed a good correlation between genotype and phenotype, but the simple virilizing and nonclassic forms showed inconsistencies between genotype and phenotype. The distribution of CYP21A2 mutations was evaluated for 21-hydroxylase deficiency patients from a single center. This study provides limited data on mutation spectrum and genotype-phenotype correlation of 21-hydroxylase deficiency in Korea.

Keywords: 21 hydroxylase deficiency, Human CYP21A2 protein, Genotype, Phenotype

\section{Introduction}

Congenital adrenal hyperplasia (CAH) is an autosomal recessive genetic disease that affects the process of cortisol synthesis in the adrenal gland. The cause of CAH in 90-95\% of affected individuals is a deficiency of 21 -hydroxylase ${ }^{1,22}$. Deficiency of this enzyme causes a reduction in cortisol and aldosterone synthesis in the adrenal gland. This results in increased secretion of adrenal corticotropic hormone (ACTH), adrenal hyperplasia, and increased synthesis of testosterone $e^{2)}$.

Based on the severity of the clinical manifestations, the 21-hydroxylase deficiency is classified into classic form, known as salt-wasting (SW) and simple virilizing (SV) type, and a nonclassic (NC) form called late-onset type. The classic form generally occurs at incidence of 1:10,000-1:15,000, while the NC form occurs in 1:1000 persons ${ }^{3}$. A higher prevalence of both classic and NC forms is found in particular races; in particular, Yupik Eskimos (Alaska) have a prevalence of 1:282, and La Reunion (France) of 1:2141 $1^{4)}$ for the classic form, while Ashkenazi Jews have a prevalence of 1:27, and Hispanics of 1:53 for the $\mathrm{NC} \mathrm{form}{ }^{5}$.

Clinical symptoms may appear at diagnosis. Symptoms that appear during the neonatal 
period in the SW form are repetitive vomiting, diarrhea, tachypnea or shortness of breath, and weight loss. Cases with severe lack of cortisol and aldosterone can show hyponatremia, hyperkalemia, dehydration, and shock in the early postnatal period $^{6,7)}$. Girls with the SV form may show ambiguous external genitalia at birth, but newborn boys show no abnormality of the external genitalia. In later years, the excessive increase in testosterone in both men and women increases the frequency of precocious puberty because of the early activation of the hypothalamic pituitary gonadal axis. Bone age (BA) also advances and short stature occurs because of the early epiphyseal fusion ${ }^{8,9)}$. The late-onset NC form is not characterized by ambiguous external genitalia at birth, but hirsutism, irregular menstruation, and other signs appear later due to the androgen excess at puberty ${ }^{10)}$.

The 21-hydroxylase genes, CYP21A2 and CYP21A1P, are located in the human leukocyte antigen (HLA) class III region of the short arm of chromosome $6 \mathrm{p} 21.3^{11)}$. The two genes are composed of 10 exons, and structurally similar. The exons are approximately 98\% identical, while the introns are $96 \%$ identical $^{1)}$ and mutations arise due to this structural similarity $^{12)}$. Intergenic recombination is associated with $95 \%$ of the 21-hydroxylase deficiency, while 5\% is associated with spontaneous mutations rather than gene conversion ${ }^{13)}$. At present, over 100 CYP21A2 mutations have been reported ${ }^{14)}$.

The purpose of this study was to evaluate endocrine patterns of patients with CAH according to their genetic mutations. We also analyzed the correlation between each phenotype and genotype.

\section{Materials and methods}

\section{Subjects}

Clinical data were collected retrospectively from a review of medical records of patients who visited the pediatric outpatient clinic at Samsung Medical Center, from November 1994 to December 2012. Twenty-seven patients (male, 19; female, 8) with $\mathrm{CAH}$ who had been diagnosed by genetic testing to have 21-hydroxylase deficiency. The Institutional Review Board of Samsung Medical Center approved this study (2013-04-065).

Data for physical examination, height, weight, electrolyte level, and hormonal level were collected from medical records. Pubertal status was assessed with the Tanner stage of breast development for girls and genital development for boys. BA was measured using the method described by Greulich and Pyle ${ }^{15)}$. Body mass index (BMI) was calculated from height and weight. The 27 patients with 21-hydroxylase deficiency were classified into the classic (SW and SV) forms and the $\mathrm{NC}$ form ${ }^{16)}$. The electrolytes were measured to confirm hyponatremia, hyperkalemia, and dehydration, while clinical findings confirmed ambiguous genitalia due to excessive androgen, precocious puberty, short stature, and hirsutism. Endocrine tests for 17-hydroxyprogesterone (17-OHP), ACTH, renin, and testosterone were performed at the time of diagnosis.
The differences in BA and chronological age (CA) ratios and BMI were investigated through follow-up period. Patients were classified according to their type of CYP21A2 mutation.

The Wizard Genomic DNA Purification Kit (Promega, Madison, WI, USA) was used to extract genomic DNA from peripheral blood samples of all patients. All exons of the CYP21A2 gene and exon-intron boundaries were amplified by polymerase chain reaction (PCR) using each primer set, and DNA was amplified using a Nucleic Acid Amplification Kit (Applied Biosystems, Foster City, CA, USA). Long PCR was performed to detect deletion or duplication by unequal meiotic crossing-over by pseudogene, and the PCR was carried by each exon. Sequence analysis was performed using an ABI Prism 3130 analyzer (Applied Biosystems). Mutations in the CYP21A2 cDNA sequence were analyzed using the reference sequences from GenBank (NM_000500.7).

\section{Statistical analysis}

The patients were divided into two groups: SW form and SV form. Each phenotype was further divided by gender. The age at diagnosis, ratio of BA to CA, BMI and hydrocortisone dose of different phenotypic groups were evaluated. The Wilcoxon signed rank test was used for between-group comparison. Statistical analyses were performed using IBM SPSS ver. 20.0 (IBM Co., Armonk, NY, USA). $P$-value of $<0.05$ was considered statistically significant.

\section{Results}

\section{Patient characteristics}

Among the 27 patients for whom follow-up observations were possible, the classic form of 21-hydroxylase deficiency was found in 26 patients. These included 15 patients (57.7\%) with the SW form (male, 11; female, 4), 11 (40.7\%) with the SV form (male, 7; female, 4), and 1 male patient (3.7\%) with the NC form. The mean follow-up observation periods were $9.00 \pm 5.17$ years for patients with the SW form, $8.38 \pm 3.68$ years for patients with the SV form, and 16.4 years for the patient with the $\mathrm{NC}$ form. The mean age at diagnosis was $0.11 \pm 0.14$ years (male, $0.13 \pm 0.16$; female, $0.05 \pm 0.04$ ) for SW form patients and $7.19 \pm 5.62$ years (male, $7.57 \pm 4.07$; female, $6.52 \pm 8.48$ ) for SV form patients. The SW form was diagnosed at a significantly earlier age in comparison with the SV form $(P=0.001)$. The male patient with the NC form was diagnosed at the age of six (Table 1).

\section{The ratio of $B A$ and $C A$ and the differences in $B M I$}

The mean value of the $\mathrm{BA}$ to $\mathrm{CA}$ ratio at diagnosis was $1.39 \pm 0.40$ (male, $1.23 \pm 0.31$; female, $1.74 \pm 0.37$ ) for the SW form patients and $1.57 \pm 0.72$ (male, $1.52 \pm 0.46$; female, $1.65 \pm 1.23$ ) for the SV form patients; these differences were not statistically significant. The final BA to CA ratio was $1.25 \pm 0.14$ (male, 
$1.26 \pm 0.16$; female, $1.24 \pm 0.11$ ) in the SW form patients and $1.23 \pm 0.19$ (male, $1.31 \pm 0.17$; female, $1.10 \pm 0.16$ ) in the SV form patients; again, the differences were not statistically significant. The boy with the $\mathrm{NC}$ form had a BA to CA ratio of 1.0 at diagnosis and a final value of 1.03 .

The mean BMI values at the last follow-up were $20.1 \pm 4.49 \mathrm{~kg} /$ $\mathrm{m}^{2}$ (male, $21.0 \pm 4.85 \mathrm{~kg} / \mathrm{m}^{2}$; female, $17.7 \pm 2.20 \mathrm{~kg} / \mathrm{m}^{2}$ ) for patients with the SW form, and $21.2 \pm 3.26 \mathrm{~kg} / \mathrm{m}^{2}$ (male, $22.6 \pm 3.87 \mathrm{~kg} / \mathrm{m}^{2}$; female, $19.8 \pm 1.09 \mathrm{~kg} / \mathrm{m}^{2}$ ) for patients with the SV form. There were no significant differences between males and females and between the SW form and SV form groups (Table 1).

\section{The changes in hormone levels}

Endocrine tests performed on the 27 patients for whom follow-up observations were possible revealed that the levels of 17-OHP, ACTH, renin, and testosterone were normalized by hydrocortisone treatment (Table 1). The hydrocortisone dosage necessary to maintain normal endocrine function in patients with the SW form was $21.7 \pm 7.6 \mathrm{mg} / \mathrm{m}^{2} /$ day for boys and $16.5 \pm 3.6 \mathrm{mg} / \mathrm{m}^{2} /$ day for girls. The hydrocortisone dosage for patients with the SV form was $15.0 \pm 5.5 \mathrm{mg} / \mathrm{m}^{2} /$ day for boys and $15.5 \pm 3.6 \mathrm{mg} / \mathrm{m}^{2} /$ day for girls, and it was $17.3 \mathrm{mg} / \mathrm{m}^{2} /$ day for the male with the $\mathrm{NC}$ form. There were no significant differences between males and females and between the SW form and SV form groups.

\section{The analysis of genes}

The analysis of 54 alleles of genes in the 27 patients for whom follow-up observations were possible revealed 13 types of mutations. The distribution of 21-hydroxylase deficiency gene mutations revealed that intron 2 splice site (c.293-13A/C > G ) mutations and large deletions were the most common, at $31.5 \%$ and $22.2 \%$ respectively, followed by p.I173N, p.R356W, and p.I172N mutations at $11.1 \%, 9.3 \%$, and $9.3 \%$, respectively. Other mutations were observed at $1.9-3.7 \%$. No novel mutations were detected (Tables 2, 3). Most patients (70.3\%) were compound heterozygotes. Homozygotes accounted for 22.2\% (6 patients, all with the SW form), and complex allele accounted for 7.4\% (2 patients only with the SV form) (Table 3).

The most common mutations in the patients with SW form were the c.293-13A/C > G and large deletion at 36.7\% and $33.3 \%$ respectively, followed by p.R356W, p.I172N, and p.I173N mutations. Among these patients, a total of 6 patients were homozygotes, accounting for 40.0\%: 2 patients had the c.293$13 \mathrm{~A} / \mathrm{C}>\mathrm{G}$ mutation, 2 patients had large deletion, 1 patient had a p.I173N mutation, and 1 patient had a $\mathrm{p} . \mathrm{R} 356 \mathrm{~W}$ mutation. The rest of the mutations were found in compound heterozygotes, but no complex alleles were detected (Tables 2, 3).

The most common mutations in the patients with SV form were c. $293-13 \mathrm{~A} / \mathrm{C}>\mathrm{G}, \mathrm{p} . \mathrm{I} 173 \mathrm{~N}$, and p.I172N, at $27.3 \%, 18.2 \%$, and $13.6 \%$ respectively, followed by large deletion, p.R356W, p.S171N, p.8bp-del, p.R484Pfs, p.L307FfsX6, and p.L306FfsX5. No homozygotes were found among these patients, but complex alleles were observed in 2 patients with large deletion $+\mathrm{c} .293$ 13A/C > G /c.293-13A/C > G+p.L306FfsX5 and p.I173N+p. Q318X+p.R357W (Tables 2, 3). The NC form patient showed large deletion and p.I172N mutations.

\section{Discussion}

$\mathrm{CAH}$ is a series of autosomal recessive diseases that produce a cortisol synthesis disorder due to enzyme deficiency imparted

Table 1. Clinical and laboratory finding of the patients with 21-hydroxylase deficiency

\begin{tabular}{|c|c|c|c|c|c|c|}
\hline \multirow{2}{*}{ Variable } & \multicolumn{2}{|c|}{ Salt wasting } & \multicolumn{2}{|c|}{ Simple virilizing } & \multicolumn{2}{|c|}{ Nonclassic } \\
\hline & Male & Female & Male & Female & Male & Female \\
\hline No. of patient & 11 & 4 & 7 & 4 & 1 & 0 \\
\hline Age at diagnosis (yr) & $0.13 \pm 0.16$ & $0.05 \pm 0.04$ & $7.57 \pm 4.07$ & $6.52 \pm 8.48$ & 6 & \\
\hline \multicolumn{7}{|l|}{$\mathrm{BA} / \mathrm{CA}$} \\
\hline Initial & $1.23 \pm 0.31$ & $1.74 \pm 0.37$ & $1.52 \pm 0.46$ & $1.65 \pm 1.23$ & 1.00 & \\
\hline Final & $1.26 \pm 0.16$ & $1.24 \pm 0.11$ & $1.31 \pm 0.17$ & $1.10 \pm 0.16$ & 1.03 & \\
\hline \multicolumn{7}{|c|}{ Body mass index $\left(\mathrm{kg} / \mathrm{m}^{2}\right)$} \\
\hline Initial & $13.50 \pm 2.17$ & $12.20 \pm 2.89$ & $17.42 \pm 3.70$ & $17.80 \pm 3.65$ & 16.64 & \\
\hline Final & $21.01 \pm 4.85$ & $17.72 \pm 2.20$ & $22.63 \pm 3.87$ & $19.81 \pm 1.09$ & 28.00 & \\
\hline \multicolumn{7}{|l|}{ 17-OHP (ng/mL) } \\
\hline Initial & 331 (30-1892) & $114(79-128)$ & $151(125-191)$ & $342(99-583)$ & 38.43 & \\
\hline Final & $13.64(0.13-72)$ & $1.52(0.72-2.34)$ & $3.02(0.13-7.72)$ & $4.84(1.91-9.73)$ & 64.00 & \\
\hline \multicolumn{7}{|l|}{ ACTH $(p g / m L)$} \\
\hline Initial & $168(16-344)$ & $601(17-1780)$ & $257(119-473)$ & $495.00(7.13-1,493.00)$ & 54.00 & \\
\hline Final & $45(7.24-158)$ & $18(18)$ & $35(18-48)$ & $47(25-71)$ & 4.83 & \\
\hline \multicolumn{7}{|l|}{ Testosterone (ng/mL) } \\
\hline Initial & $0.83(0.04-3.32)$ & $11(0.74-20)$ & $3.33(1.24-5.23)$ & $1.03(0.94-1.01)$ & 1.67 & \\
\hline Final & $0.62(0.01-2.91)$ & $0.13(0.13)$ & $2.54(0.01-3.20)$ & $0.07(0.01-0.12)$ & 4.28 & \\
\hline Renin (ng/mL), initial & $32.00(8.40-72.00)$ & $34(34)$ & $19(16-21)$ & $8.34(8.20-8.53)$ & 6.75 & \\
\hline
\end{tabular}

Values are presented as mean \pm standard deviation or median (range).

BA, bone age; CA, chronological age; 17-OHP, 17-hydroxyprogesterone; ACTH, adrenocorticotropic hormone. 
by gene mutations that include cytochrome P450c21. The result is adrenal hyperplasia and an excessive accumulation of cortisol precursors and androgen. Several clinical findings,

Table 2. Phenotype and genotype of 27 patients with 21 hydroxylase deficiency

\begin{tabular}{|c|c|c|c|}
\hline Patient & Sex & Phenotype & Genotype \\
\hline 1 & Male & SW & c.293-13A/C>G/c.293-13A/C>G \\
\hline 2 & Female & SW & c. $293-13 A / C>G / c .293-13 A / C>G$ \\
\hline 3 & Male & SW & c.293-13A/C>G/8-bp del \\
\hline 4 & Male & SW & c.293-13A/C>G/large deletion \\
\hline 5 & Male & SW & c.293-13A/C>G/large deletion \\
\hline 6 & Male & SW & c.293-13A/C>G/p.R356W \\
\hline 7 & Male & SW & Large deletion/large deletion \\
\hline 8 & Male & SW & Large deletion/large deletion \\
\hline 9 & Male & SW & Large deletion/c.293-13A/C>G \\
\hline 10 & Male & SW & Large deletion/c.293-13A/C>G \\
\hline 11 & Female & SW & Large deletion/conversion \\
\hline 12 & Male & SW & p.1172N/large deletion \\
\hline 13 & Female & SW & p.1173N/p.1173N \\
\hline 14 & Male & SW & p.R356W/p.R356W \\
\hline 15 & Male & SW & c.293-13A/C>G/p.R356W \\
\hline 16 & Male & SV & $\begin{array}{l}\text { Large deletion+c.293-13A/C>G/ } \\
\text { c.293-13A/C>G+p.L306FfsX5 }\end{array}$ \\
\hline 17 & Male & SV & Large deletion/p.R356W \\
\hline 18 & Male & SV & c.293-13A/C>G/p.1173N \\
\hline 19 & Male & SV & c.293-13A/C>G/p.R484Pfs \\
\hline 20 & Male & SV & c.293-13A/C>G/p.S171N \\
\hline 21 & Female & SV & c.293-13A/C>G/p.8-bp del \\
\hline 22 & Male & SV & p.1172N/p.L307FfsX6 \\
\hline 23 & Female & SV & p.1172N/p.L306FfsX5 \\
\hline 24 & Female & SV & p. $1172 \mathrm{~N} / \mathrm{c} .293-13 \mathrm{~A} / \mathrm{C}>\mathrm{G}$ \\
\hline 25 & Female & SV & p. $.173 N / p .1173 N$ \\
\hline 26 & Male & SV & p.1173N+p.Q318X+p.R357W \\
\hline 27 & Male & NC & Large deletion/p.I172N \\
\hline
\end{tabular}

SW, salt wasting; SV, simple virilizing; NC, nonclassic; 8-bp del, 8-bp deletion. such as virilization of external genitalia and loss of salt, occur in response to intermediary metabolites ${ }^{16}$. To date, seven types of enzyme deficiency have been identified: 21-hydroxylase, $11 \beta$-hydroxylase, $3 \beta$-hydroxysteroid dehydrogenase, 17 $\alpha$-hydroxylase/17,20-lyase, and 18-hydroxylase. Among these, 21-hydroxylase deficiency accounts for $90-95 \%$ of this disorder ${ }^{13,16)}$.

The 21-hydroxylase gene is composed of $C Y P 21 A 2$, a functional gene, and $C Y P 21 A 1 P$, a nonfunctional gene. The gene is arranged beside genes that encode complement factors called $\mathrm{C} 4 \mathrm{~A}$ and $\mathrm{C} 4 \mathrm{~B}$ and it consists of a tandem repeat composed of $C 4 / C Y P 21 A 2$ and $C 4 / C Y P 21 A 1 P^{11)}$. Since $C Y P 21 A 2$ and $C Y P 21 A 1 P$ are structurally similar, $98 \%$ of exons are identical, and $96 \%$ of introns are identical ${ }^{1)}$. Therefore, due to the structural similarity of these duplicated regions, misalignment occurs during meiosis, resulting in mutations ${ }^{12)}$. Gene conversion accounts for $75 \%$ of intergenic recombination, which occurs via transfer to $C Y P 21 A 2$ during mitosis ${ }^{17,18)}$. Mutations such as deletions, CYP21A2/CYP21A1P chimeric genes, and duplication during meiotic crossover of $C Y P 21 A 2$ account for the remaining $20-25 \%{ }^{19)}$.

The correlation between 21-hydroxylase genotype and phenotype has been studied in a variety of ethnic groups ${ }^{7,12,20)}$. The 21-hydroxylase gene mutations are classified into 3 groups according to genotype and phenotype based on enzymatic activity $^{7,12,20)}$. Group A is the SW form, which lacks enzyme activity and includes deletion, p.Q318X, and p.R356W, and c.293-13A/C > G mutations. Group B is the SV form, with enzymatic activity of $1-5 \%$, and is mainly a p.I172N mutation ${ }^{21)}$. Group C is the NC form, with enzyme activity of $20-50 \%$, and

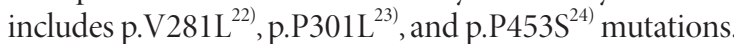

Early diagnosis of this disease can occur for the SW form, which shows acute symptoms at a relatively early age, and for girls with SV, who show external genital abnormality at birth. However, no striking symptoms are observed in boys with the SV form, so diagnosis is often delayed, as seen in previous reports ${ }^{25)}$. Likewise, this study also revealed a significant

Table 3. Allelic frequency of CYP21A2 mutation in patients with 21-hydroxylase deficiency

\begin{tabular}{|c|c|c|c|c|}
\hline Mutation & Salt wasting & Simple virilizing & Nonclassic & Total \\
\hline c.293-13A/C>G & $11(36.7)$ & $6(27.3)$ & $0(0)$ & $17(31.5)$ \\
\hline Large deletion & $10(33.3)$ & $1(4.5)$ & $1(50.0)$ & $12(22.2)$ \\
\hline p.R356W (c.1066C>T) & $4(13.3)$ & $1(4.5)$ & $0(0)$ & $5(9.3)$ \\
\hline p.S171N (c.512T>A) & $0(0)$ & $1(4.5)$ & $0(0)$ & $1(1.9)$ \\
\hline p.I172N (c.515T>A) & $1(3.3)$ & $3(13.6)$ & $1(50.0)$ & $5(9.3)$ \\
\hline p. $1173 \mathrm{~N}(\mathrm{c} .518 \mathrm{~T}>\mathrm{A})$ & $2(6.6)$ & $4(18.2)$ & $0(0)$ & $6(11.1)$ \\
\hline Conversion & $1(3.3)$ & $0(0)$ & $0(0)$ & $1(1.9)$ \\
\hline 8-bp deletion (c.329_336delGAGACTAC) & $1(3.3)$ & $1(4.5)$ & $0(0)$ & $2(3.7)$ \\
\hline p.R484Pfs (c.1451dupC) & $0(0)$ & $1(4.5)$ & $0(0)$ & $1(1.9)$ \\
\hline p.L307FfsX6 (c.920_921insT) & $0(0)$ & $1(4.5)$ & $0(0)$ & $1(1.9)$ \\
\hline p.L306FfsX5 (c.920dupT) & $0(0)$ & $1(4.5)$ & $0(0)$ & $1(1.9)$ \\
\hline Large deletion+c.293-13A/C>G+p.L306FfsX5 (c.920dupT) & $0(0)$ & $1(4.5)$ & $0(0)$ & $1(1.9)$ \\
\hline p.Q318X+p.R357W (c.955C>T+c.1069C>T) & $0(0)$ & $1(4.5)$ & $0(0)$ & $1(1.9)$ \\
\hline Total & $30(100)$ & $22(100)$ & $2(100)$ & $54(100)$ \\
\hline
\end{tabular}

Values are presented as number (\%). 
difference in mean age at diagnosis, at $0.11 \pm 0.14$ years for the SW form and 7.19 \pm 5.62 years for the SV form.

Patients with the SW or SV form usually show a shorter final adult height than the average adult height, of approximately 1-2 SD (standard deviation) ${ }^{16,25)}$. The reasons are considered to be the increased rate of bone maturation and early epiphyseal maturation due to excessive secretion of androgens, the promotion of bone maturity and epiphyseal maturation caused by precocious puberty, and the reduction in the pubertal growth time ${ }^{26)}$. In this study, the BA to CA ratio increased in both the SW form and SV form, with no significant difference between the forms of the disease. Numerous reports ${ }^{27)}$ indicate that patients who received appropriate treatment showed a higher risk of obesity and greater increases in BMI. The mean BMI in each group was lower than $25 \mathrm{~kg} / \mathrm{m}^{2}$ which means there is no tendency toward obesity. Other recent studies have also reported no significant differences in BMI among patients with different phenotypes. Therefore, BMI cannot be considered a measure of the complications of disease ${ }^{28)}$.

Wilkins et al. ${ }^{29)}$ and Batter et al. ${ }^{30)}$ demonstrated in 1950 that cortisone is an effective treatment for $\mathrm{CAH}$, and the combined use of cortisone and mineralocorticoid has greatly lowered the mortality and morbidity rates and increased the life expectancy of these patiens. The treatment of this disease is aimed at reducing the excessive secretion of $\mathrm{ACTH}$ and replenishing mineralocorticoid levels. The preferred drug in substitution therapy for glucocortocoid is hydrocortisone, which is administered at $10-20 \mathrm{mg} / \mathrm{m}^{2} /$ day three times. In this study, hydrocortisone was administered at $17.2 \mathrm{mg} / \mathrm{m}^{2} /$ day on average, and the administered doses did not differ significantly between phenotypes or genders.

Recently, Choi et al. ${ }^{31)}$ reported a genotype-phenotype correlation of 21-hydroxylase deficient patients in Korea. They concluded that the overall concordance between genotype and phenotype was $96.8 \%$ that is consistent with previous reports ${ }^{19,32,33}$. In this study, each mutation were divided into three groups to correlate their genotypes, positive predictive value in $83.3 \%, 40.9 \%$ with SW, SV, respectively. No gene mutation was associated with the patient with the NC form. As a result, there were the difference between the previous reports and a genotype-phenotype correlation. The mutations of gene causing 21-hydroxylase deficiency in Koreans reported in domestic research were observed in the sequence of c.293-13A/ $\mathrm{C}>\mathrm{G}(23 \%)$, deletion/large conversion (18\%), p.I172N (11\%), p.Q318X (9.3\%), p.R356W $(8.0 \%)^{34,35)}$. Overseas reports indicate the main mutations to be deletion, c.293-13A/C > G, p.I172N, p.R356W, and p.Q318X ${ }^{2)}$. In a study of Korean, the frequency of the deletion of the CYP21A2 gene was $18.0 \%$, Japan and China were $12.0 \%, 4.7 \%$, respectively ${ }^{36,37)}$. That is lower than in Western countries at $20-30 \%^{7,12)}$.

The frequencies of gene mutations causing 21-hydroxylase deficiency in this study showed discrepancy from the numerical values in other domestic and overseas reports, but the common mutation types were consistent, with c.293-13A/C > G, large deletion, p.R356W, p.I172N, and p.I173N accounting for most of the mutations. The CYP21A2 mutation genotypes and phenotypes vary according to the severity of disease, and this also showed a good correlation ${ }^{2)}$. The c.293-13A/C $>$ G, deletion, and p.R356W mutations are as associated with the SW form $^{38)}$, while c.293-13A/C $>$ G is known to cause both the SW and SV forms ${ }^{39)}$. p.V281L is mainly found in the $\mathrm{NC}$ form ${ }^{21)}$.

In conclusion, the mutations of the CYP21A2 gene in the SW, $\mathrm{SV}$, and $\mathrm{NC}$ forms of CAH were confirmed in 27 patients in a single center for whom follow-up observations were possible. The analysis of 54 alleles revealed 13 types of mutation. The SW form showed a good correlation between genotype and phenotype, but the SV and NC forms showed inconsistencies between genotype and phenotype. The SW and SV forms showed large deletion, p.I172N, and p.I173N mutations, while the $\mathrm{NC}$ form showed large deletion and p.I172N mutations.

The distribution of CYP21A2 mutations was evaluated for 21-hydroxylase deficiency patients from a single center. This study provides limited data on mutation spectrum and genotype-phenotype correlation of 21-hydroxylase deficiency in Korea.

\section{Conflict of interest}

No potential conflict of interest relevant to this article was reported.

\section{References}

1. Krone N, Arlt W. Genetics of congenital adrenal hyperplasia. Best Pract Res Clin Endocrinol Metab 2009;23:181-92.

2. White PC, Speiser PW. Congenital adrenal hyperplasia due to 21-hydroxylase deficiency. Endocr Rev 2000;21:245-91.

3. Trakakis E, Basios G, Trompoukis P, Labos G, Grammatikakis I, Kassanos D. An update to 21-hydroxylase deficient congenital adrenal hyperplasia. Gynecol Endocrinol 2010;26:63-71.

4. Pang S, Clark A. Newborn screening, prenatal diagnosis, and prenatal treatment of congenital adrenal hyperplasia due to 21-hydroxylase deficiency. Trends Endocrinol Metab 1990;1:300-7.

5. Speiser PW, Dupont B, Rubinstein P, Piazza A, Kastelan A, New MI. High frequency of nonclassical steroid 21-hydroxylase deficiency. Am J Hum Genet 1985;37:65067.

6. Raiti S, Newns GH. Congenital adrenal hyperplasia. Arch Dis Child 1964;39:324-33.

7. Speiser PW, Dupont J, Zhu D, Serrat J, Buegeleisen M, TusieLuna MT, et al. Disease expression and molecular genotype in congenital adrenal hyperplasia due to 21-hydroxylase deficiency. J Clin Invest 1992;90:584-95.

8. New MI. An update of congenital adrenal hyperplasia. Ann N Y Acad Sci 2004;1038:14-43.

9. Kaplan SA. Diseases of the adrenal cortex: II. Congenital adrenal hyperplasia. Pediatr Clin North Am 1979;26:77-89. 
10. Speiser PW. Nonclassic adrenal hyperplasia. Rev Endocr Metab Disord 2009; 10:77-82.

11. White PC, Grossberger D, Onufer BJ, Chaplin DD, New MI, Dupont B, et al. Two genes encoding steroid 21-hydroxylase are located near the genes encoding the fourth component of complement in man. Proc Natl Acad Sci U S A 1985;82:1089-93.

12. Wedell A, Thilen A, Ritzen EM, Stengler B, Luthman H. Mutational spectrum of the steroid 21-hydroxylase gene in Sweden: implications for genetic diagnosis and association with disease manifestation. J Clin Endocrinol Metab 1994;78:1145-52.

13. Lee HH. CYP21 mutations and congenital adrenal hyperplasia. Clin Genet 2001;59:293-301.

14. The Human Cytochrome P450(CYP) Allele Nomenclature Committee. CYP21A2: CYP 21A1 allele nomenclature [Internet]. Stockholm: The Human Cytochrome P450(CYP) Allele Nomenclature Committee; c2013 [cited 2013 Aug 19]. Available from: http://www.cypalleles.ki.se/ cyp21.htm.

15. Greulich WW, Pyle SI. Radiographic atlas of skeletal development of hand and wrist. 2nd ed. Redwood City: Stanford University Press, 1959.

16. Speiser PW, White PC. Congenital adrenal hyperplasia. N Engl J Med 2003;349:776-88.

17. Higashi Y, Yoshioka H, Yamane M, Gotoh O, FujiiKuriyama Y. Complete nucleotide sequence of two steroid 21-hydroxylase genes tandemly arranged in human chromosome: a pseudogene and a genuine gene. Proc Natl Acad Sci U S A 1986;83:2841-5.

18. Tusie-Luna MT, White PC. Gene conversions and unequal crossovers between CYP21 (steroid 21-hydroxylase gene) and CYP21P involve different mechanisms. Proc Natl Acad Sci U S A 1995;92:10796-800.

19. Parajes S, Quinteiro C, Dominguez F, Loidi L. High frequency of copy number variations and sequence variants at CYP21A2 locus: implication for the genetic diagnosis of 21-hydroxylase deficiency. PLoS One 2008;3:e2138.

20. Higashi Y, Hiromasa T, Tanae A, Miki T, Nakura J, Kondo T, et al. Effects of individual mutations in the P-450(C21) pseudogene on the $\mathrm{P}-450(\mathrm{C} 21)$ activity and their distribution in the patient genomes of congenital steroid 21-hydroxylase deficiency. J Biochem 1991;109:638-44.

21. Amor M, Parker KL, Globerman H, New MI, White PC. Mutation in the CYP21B gene (Ile-172: Asn) causes steroid 21-hydroxylase deficiency. Proc Natl Acad Sci U S A 1988;85:1600-4.

22. Speiser PW, New MI. Genotype and hormonal phenotype in nonclassical 21-hydroxylase deficiency. J Clin Endocrinol Metab 1987;64:86-91.

23. Tusie-Luna MT, Speiser PW, Dumic M, New MI, White PC. A mutation (Pro-30 to Leu) in CYP21 represents a potential nonclassic steroid 21-hydroxylase deficiency allele. Mol Endocrinol 1991;5:685-92.

24. Nikoshkov A, Lajic S, Holst M, Wedell A, Luthman H.
Synergistic effect of partially inactivating mutations in steroid 21-hydroxylase deficiency. J Clin Endocrinol Metab 1997;82:194-9.

25. New MI, Gertner JM, Speiser PW, del Balzo P. Growth and final height in classical and nonclassical 21-hydroxylase deficiency. Acta Paediatr Jpn 1988;30 Suppl:79-88.

26. Soliman AT, AlLamki M, AlSalmi I, Asfour M. Congenital adrenal hyperplasia complicated by central precocious puberty: linear growth during infancy and treatment with gonadotropin-releasing hormone analog. Metabolism 1997;46:513-7.

27. Cornean RE, Hindmarsh PC, Brook CG. Obesity in 21-hydroxylase deficient patients. Arch Dis Child 1998;78:261-3

28. Manoli I, Kanaka-Gantenbein Ch, Voutetakis A, ManiatiChristidi M, Dacou-Voutetakis C. Early growth, pubertal development, body mass index and final height of patients with congenital adrenal hyperplasia: factors influencing the outcome. Clin Endocrinol (Oxf) 2002;57:669-76.

29. Wilkins L, Lewis RA, Klein R, Rosemberg E. The suppression of androgen secretion by cortisone in a case of congenital adrenal hyperplasia. Bull Johns Hopkins Hosp 1950;86:249-52.

30. Bartter FC, Forbes AP, Leaf A. Congenital adrenal hyperplasia associated with the adrenogenital syndrome: an attempt to correct its disordered hormonal pattern. J Clin Invest 1950;29:797.

31. Choi JH, Jin HY, Lee BH, Ko JM, Lee JJ, Kim GH, et al. Clinical phenotype and mutation spectrum of the CYP21A2 gene in patients with steroid 21-hydroxylase deficiency. Exp Clin Endocrinol Diabetes 2012;120:23-7.

32. Finkielstain GP, Chen W, Mehta SP, Fujimura FK, Hanna RM, Van Ryzin C, et al. Comprehensive genetic analysis of 182 unrelated families with congenital adrenal hyperplasia due to 21-hydroxylase deficiency. J Clin Endocrinol Metab 2011;96:E161-72.

33. Friaes A, Rego AT, Aragues JM, Moura LF, Mirante A, Mascarenhas MR, et al. CYP21A2 mutations in Portuguese patients with congenital adrenal hyperplasia: identification of two novel mutations and characterization of four different partial gene conversions. Mol Genet Metab 2006;88:58-65

34. Ko JM, Choi JH, Kim GH, Yoo HW. Genotype of steroid 21-hydroxylase gene and clinical characteristics in patients with congenital adrenal hyperplasia 21-hydroxylase deficiency. J Korean Soc Pediatr Endocrinol 2005;10:57-63.

35. Jin DK, Kim JS, Song SM, Park SJ, Hwang HZ, On HY, et al. A study on the relationship between genotype and phenotype in korean patients with congenital adrenogenital syndrome caused by 21-hydroxylase deficiency. J Korean Soc Endocrinol 2000;15:237-47.

36. Asanuma A, Ohura T, Ogawa E, Sato S, Igarashi Y, Matsubara Y, et al. Molecular analysis of Japanese patients with steroid 21-hydroxylase deficiency. J Hum Genet 1999;44:312-7. 
37. Lee HH, Lee YJ, Wang YM, Chao HT, Niu DM, Chao $\mathrm{MC}$, et al. Low frequency of the CYP21A2 deletion in ethnic Chinese (Taiwanese) patients with 21-hydroxylase deficiency. Mol Genet Metab 2008;93:450-7.

38. Lee HH, Chao HT, Ng HT, Choo KB. Direct molecular diagnosis of CYP21 mutations in congenital adrenal hyperplasia. J Med Genet 1996;33:371-5.
39. Schulze E, Scharer G, Rogatzki A, Priebe L, Lewicka $S$, Bettendorf $M$, et al. Divergence between genotype and phenotype in relatives of patients with the intron 2 mutation of steroid-21-hydroxylase. Endocr Res 1995;21:359-64. 